\section{Online MD Pediatrics Exit Examination: A Novel Method of End Curriculum Summative Assessment Amidst the Ongoing COVID-19 Pandemic}

Due to the situation arising from the ongoing coronavirus disease-19 (COVID-19) pandemic, the erstwhile Medical Council of India (MCI), in its circular of May 22, 2020, relaxed the norms for appointment of external examiners in postgraduate examination and permitted partial online examinations. We recently successfully conducted the MD Pediatrics exit examinations online, and wish to share our experience.

All theory papers, practical/clinical, objective structured clinical examinations (OSCEs), and oral/viva-voce examination were conducted in early-May, 2020 with strict observance of social distancing and other measures to prevent COVID-19 transmission. Although the theory examination (clinical vignettes based) did not pose much challenge, conducting the practical examination in compliance with the norms was an uphill task. Two external examiners were in the examination board to conduct and supervise the practical examination on the online video platform Skype. A mixed approach was followed in which both the internal examiner (physically present in the examination room) and external examiners (online) were involved in assessment by face-to-face, real-time online evaluation. Every possible effort was made to include all essential components from a traditional practical exit examination, including clinical cases, OSCEs, spot cases, and oral/viva voce examination.

Practical examination was conducted over two days under real-time video monitoring, supervision, and active participation by external examiners. Two desktop system having inbuilt camera with high speed LAN connection were setup in pediatric ward and NICU. A Skype group consisting of all examiners was created. Pre-examination meetings on skype involving all examiners were conducted wherein examination components (OSCE, spot cases, short and long cases) and strategy was discussed beforehand. The OSCEs were designed to test all six domains of the Accreditation Council for Graduate Medical Education (ACGME) [1]. On the day of examination, case presentation by examinees was done in the traditional way except that the external examiners were directing the examination process online, in real time and virtually. Assesment sheets and final marks sheet were shared and signed by e-mail. Both the external examiners provided positive feedback on the overall examination process and logistics. The overall expenses were less than one-third of the conventional physical examination method.

Computer-based online testing in a high-stakes examination of the medical curriculum is one of the best ways to test the clinical skills of an outgoing postgraduate, and in the current times of COVID-19 pandemic it seems to be a feasible option in order to meet the timeline of course completion [2]. Dearth of clinical material due to COVID-19 lockdown may be addressed by mock clinical situations, virtual case scenarios and high fidelity mannequins.

Skype, Zoom, Webex meetings, Google Meet, Team Viewer, and various other web conferencing platforms have taken over medical education through webinars and online teaching sessions during the COVID-19 pandemic, as face-to-face classes are suspended in almost all places [3]. Boeve, et al. [2] demonstrated a similar score of students in the computer-based examination, as compared to paper-based examination and comparable acceptance by teachers and students in the medical curriculum. The USMLE and MRCPCH examinations are being conducted using online testing methods since long.

Acceptance by examinees and examiners, appreciation of clinical findings by external examiners, need for extra software/ hardware, technical glitches, communication errors and other institutional barriers are challenges to be considered while conducting such an examination. Mock examinations, preexamination meeting and briefing of examiners conveying case details, and support from the information technology department helped in overcoming these challenges.

Prateek Kumar Panda, ${ }^{1 *}$ Nowneet Kumar Bhat ${ }^{1}$ and RAVIKANT $^{2}$

From ${ }^{1}$ Department of Pediatrics, and ${ }^{2}$ Director and CEO, All India Institute of Medical Sciences, Rishikesh, India. *drprateekpanda@gmail.com

\section{REFERENCES}

1. Witteles RM, Verghese A. Accreditation Council for Graduate Medical Education (ACGME) Milestones - Time for a revolt? JAMA Intern Med. 2016;176:1599-600.

2. Boevé AJ, Meijer RR, Albers CJ, Beetsma Y, Bosker RJ. Introducing computer-based testing in high-stakes examinations in higher education:Results of a field experiment. PLoS One. 2015;10:e0143616.

3. Ferrel MN, Ryan JJ. The Impact of COVID-19 on medical education. Cureus. 2020;12:e7492. 